\title{
Tactile Object Familiarity in the Blind Brain Reveals the Supramodal Perceptual-Mnemonic Nature of the Perirhinal Cortex
}

\author{
Laura Cacciamani* and Lora T. Likova \\ The Smith-Kettlewell Eye Research Institute, San Francisco, CA, USA
}

This study is the first to investigate the neural underpinnings of tactile object familiarity in the blind during both perception and memory. In the sighted, the perirhinal cortex (PRC) has been implicated in the assessment of visual object familiarity - a crucial everyday task - as evidenced by reduced activation when an object becomes familiar. Here, to examine the PRC's role in tactile object familiarity in the absence of vision, we trained blind participants on a unique memory-guided drawing technique and measured brain activity while they perceptually explored raised-line drawings, drew them from tactile memory, and scribbled (control). Functional magnetic resonance imaging (fMRI) before and after a week of training revealed a significant decrease in PRC activation from preto post-training (i.e., from unfamiliar to familiar) during perceptual exploration as well

OPEN ACCESS

Edited by:

Carol Seger

Colorado State University, USA

Reviewed by:

James W. Lewis,

West Virginia University, USA Chris B. Martin,

University of Western Ontario, Canada Wei-Chun Wang,

Duke University, USA

*Correspondence:

Laura Cacciamani Icacciamani@gmail.com

Received: 07 November 2015

Accepted: 22 February 2016

Published: 12 April 2016

Citation:

Cacciamani L and Likova LT (2016) Tactile Object Familiarity in the Blind

Brain Reveals the Supramodal Perceptual-Mnemonic Nature of the Perirhinal Cortex.

Front. Hum. Neurosci. 10:92. doi: 10.3389/fnhum.2016.00092 as memory-guided drawing, but not scribbling. This familiarity-based reduction is the first evidence that the PRC represents tactile object familiarity in the blind. Furthermore, the finding of this effect during both tactile perception and tactile memory provides the critical link in establishing the PRC as a structure whose representations are supramodal for both perception and memory.

Keywords: blindness, familiarity, perirhinal cortex, tactile memory, tactile perception

\section{INTRODUCTION}

The ability to use the sense of vision to perceive objects in the environment seems instantaneous and effortless and therefore is often taken for granted. Without vision, though, perceiving even the simplest of objects suddenly becomes challenging. Individuals who are blind must rely on other senses, such as their sense of touch, in order to serially explore and understand the world around them. One task that is particularly important to any daily environmental interaction is the ability to assess whether a confronted object is familiar such that an appropriate response to that object can be formed. By "familiar," we mean that the object has been encountered previously such that stored meaningful object memories exist and can be accessed, thereby making the object recognizable. While a wealth of previous work has investigated this ability to assess object familiarity in the sighted, an understanding of the neural underpinnings of tactile object familiarity in the blind is lacking. The current study provides the first investigation of tactile object familiarity representations in the blind brain.

A key neural structure known to be involved in object familiarity-at least in the visual domain -is the perirhinal cortex (PRC), which lies near the hippocampus in the medial temporal 
lobe (MTL). The PRC traditionally has been implicated in memory-particularly in the recognition of familiar objects (Brown and Aggleton, 2001). Its involvement in recognition memory is typically evidenced not by an increase but by a reduction in neuronal responses to visual stimuli that have been encountered previously, thereby rendering them familiar. This familiarity-based response reduction has been observed in single neurons via electrophysiological recordings in monkeys and rats (Ringo, 1996; Brown and Xiang, 1998; Suzuki and Eichenbaum, 2000) as well as via blood oxygen level dependent (BOLD) fMRI in human neuroimaging studies (Henson et al., 2003). Further support for the PRC's role in visual object familiarity comes from studies showing that damage to or surgical removal of the PRC in monkeys, rats, and humans impairs recognition memory for individual objects (Zola-Morgan et al., 1989; Meunier et al., 1993; Suzuki et al., 1993; Mumby and Pinel, 1994; Buffalo et al., 1998). Moreover, assessing object familiarity seems to be a role specifically associated with the PRC rather than other MTL structures. The hippocampus, for instance, represents the relative spatial locations of objects rather than their relative familiarity (Brown and Aggleton, 2001). The entorhinal cortex (ERC), which others have posited might operate as a bridge between the hippocampus and PRC, signals both object familiarity and spatial location, however, not as strongly as either the PRC or the hippocampus, respectively. Indeed, lesions to the ERC have less severe effects on object recognition abilities than lesions to the PRC (Meunier et al., 1993).

The increased interest in the PRC has led to an abundance of recent work revealing that the PRC's familiarity representations transcend visual recognition memory and also subserve visual perception (Murray and Bussey, 1999; Bussey et al., 2002, 2005; Murray et al., 2007; Baxter, 2009; Peterson et al., 2012; Nadel and Peterson, 2013). For instance, research in rats, monkeys, and humans has shown that the PRC is involved in the visual discrimination of simultaneously presented complex objects when working memory demands are low (Bussey et al., 2002; Lee et al., 2005; Barense et al., 2007; Bartko et al., 2007). This type of simultaneous object discrimination task has also been used to demonstrate the PRC's role in perceptual tasks that require access to familiarity representations in particular. For example, when rats are simultaneously presented with two objects-one familiar and one novel-and are allowed to freely explore them, the typical finding is a preference for exploring the novel item-a result indicating the animal's ability to visually assess and distinguish object familiarity (Ennaceur and Delacour, 1988). Selective damage to the PRC via lesions or chemical antagonists has been shown to impair this discrimination ability such that the rat no longer exhibits a novelty preference, and rather, explores the familiar and novel item equally (Ennaceur et al., 1996; Ennaceur and Aggleton, 1997; Abe et al., 2004). This important work has shown that the PRC is necessary in the visual discrimination of item familiarity.

From the above research, the key involvement of the PRC in visual object familiarity during both perceptual and memory tasks is apparent, thus indicating its perceptual-mnemonic functioning. While this previous work has been conducted in the visual modality, other prior research suggests that the PRC's representations are not unimodal. Given its position at the culmination of the occipitotemporal object-processing stream, the PRC receives input from all sensory modalities (Suzuki and Amaral, 1994), thereby implicating it as a site suitable for cross-modal integration (Murray and Richmond, 2001; Taylor et al., 2006). Indeed, individual neurons in the PRC have been shown to respond to multimodal (both visual and auditory) input (Desimone and Gross, 1979). Neuroimaging studies in humans have shown that the PRC's representations are stronger during an object-matching task when the input is cross-modal (visual-tactile) vs. unimodal (visual-visual or tactile-tactile) (Holdstock et al., 2009). Furthermore, damage to the PRC results in impaired auditory familiarity assessment during recognition memory tests in sighted humans (Martin et al., 2011). Similarly, in sighted primates, selective lesions to the PRC impair not only visual but also tactile recognition memory (Suzuki et al., 1993), further suggesting that the PRC's mnemonic representations are multimodal, at least in the sighted. Note, however, that these tactile impairments arising from PRC lesions were found only for memory tasks, while tactile perceptual discrimination was unaffected (Suzuki et al., 1993) - an important distinction considering the PRC's involvement in not only memory but also perception in the visual modality. Thus, it remains unclear whether the 'dual' - perceptual and mnemonic - nature of familiarity assessment in the PRC extends beyond the visual modality, and in particular, to the tactile modality. Furthermore, these studies investigated the cross-modal nature of the PRC only in sighted participants. No prior studies have investigated tactile object familiarity in the PRC in blind individuals, including those who have never been able to use vision to form object representations.

The current study aims to fill these gaps in the literature by assessing tactile representations of object familiarity in the PRC in blind individuals. Moreover, we aim to determine for the first time whether the PRC's tactile familiarity representations in the blind are involved in just memory or also perception. Doing so will provide the missing link in establishing the PRC as not just a perceptual-mnemonic structure, but also one that functions independently of sensory modality and visual experience. Furthermore, this study will provide the field's first glimpse into tactile familiarity in the blind brain-an important contribution that will lay the foundation necessary for future tactile rehabilitation research.

While no previous work has investigated the blind PRC in particular, evidence of cortical reorganization of the blind brain toward representing tactile information in other brain areas traditionally involved in vision does exist (Amedi et al., 2002; Pascual-Leone et al., 2005; Sathian and Stilla, 2010; Likova, 2010, 2012, 2013). Likova (2010, 2012, 2013) for instance, conducted various studies examining training-based reorganization in the primary "visual" cortex (V1) in the blind as a function of learning. In these studies, blind participants underwent a special training on a demanding tactile memory paradigm (Figure 1A), which we employ in the current study as well. In this paradigm, participants learned how to 


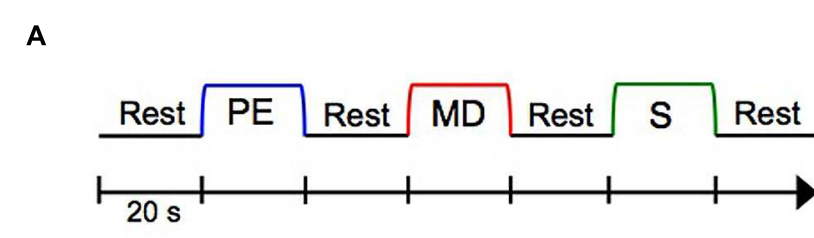

B
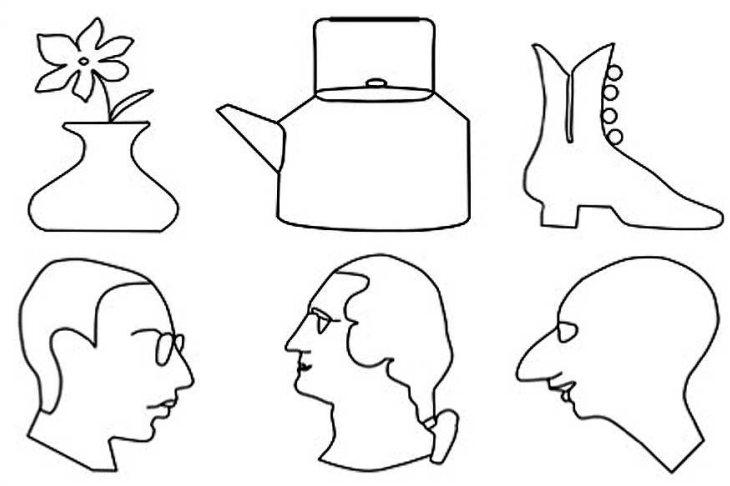

C

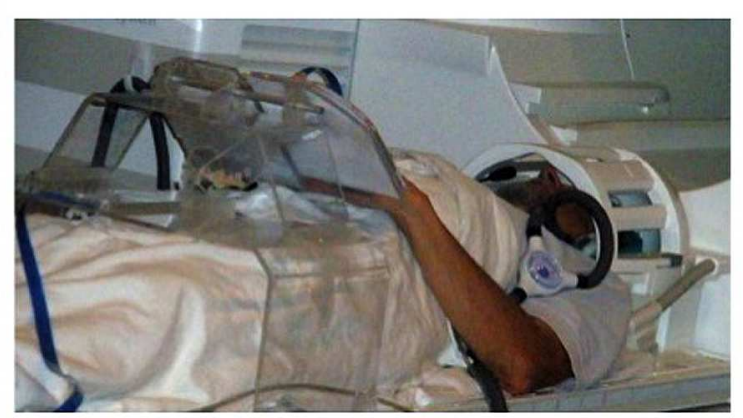

FIGURE 1 | The experimental functional magnetic resonance imaging (fMRI) design, stimuli, and setup. (A) Three-task blocked experimental design, repeated twelve times (twice per stimulus). Auditory cues signaled the beginning of each block. (B) Raised line-drawing stimuli. (C) A participant operating our multimodal MRI-compatible drawing device. PE, Perceptual Exploration; MD, Memory Drawing; S, Scribble.

memorize with high precision raised-line drawings (Figure 1B) while exploring them one at a time with their left hand (Perceptual Exploration, or PE, condition), and how to use that memory representation for drawing the stimulus from memory using their right hand (Memory Drawing, or MD, condition). As a motor control task, participants also drew randomly with their right hand (Scribble, or S, condition). The use of different hands for exploration and drawing required participants to rely on tactile memory rather than motor representations, rendering this non-visual drawing task even more difficult. Over 5 days, participants were trained on this task, such that by the end of training, participants' drawings improved substantially. Before and after training, whole-brain fMRI scans were conducted in order to examine the effect of training on cortical reorganization (Figure 1C). Likova (2013, 2014) found that only a week of this "CognitiveKinesthetic Training" resulted in dramatic changes in the primary visual area V1, consistent across different categories of visual deprivation-congenitally blind, late-onset blind, and blindfolded. BOLD response waveforms in V1 went from being immature and erratic to well-formed and closely fit to the BOLD predictor for the memory-guided drawing task (Likova, 2012). This pre- to post-training difference in activation was not observed in a control area, the motor cortex (M1). This remarkable finding demonstrates that areas typically involved in vision can reorganize to represent tactile memory information in the blind after only 1 week of well-targeted training. The results implicated V1 as the implementation of the theoretical 'visuo-spatial sketchpad' for working memory in humans and led to its reconceptualization into a 'modalityindependent (or supramodal) spatial sketchpad' (Likova, 2012, 2013). Furthermore, this drawing training paradigm has the dualadvantage of being both a non-visual and a causal intervention. Thus, it made it possible to reveal for the first time complex patterns of training-based encoding/retrieval reorganization in the inferotemporal cortex and the hippocampus (Likova, 2015), which has important implications for the emerging view of perception/memory interactions and their dynamics through the learning process.

In the present study, we employed Likova's CognitiveKinesthetic Training (Likova, 2010, 2012, 2013, 2014) to assess perceptual-mnemonic representations of tactile object familiarity in the blind PRC. Participants were trained 2 hours per day for 5 days on how to perceptually explore (PE) the raisedline drawings and subsequently draw them from tactile memory (MD). Although the line drawings depicted well-known, realworld objects, they were unrecognizable, meaningless, and therefore "unfamiliar" to the blind participants before training. After learning these line drawings as a result of the training, object memories were formed such that the line drawings became recognizable, meaningful, and therefore "familiar" to our blind participants after training. Using BOLD fMRI, we compared PRC activation before training (when the objects were unfamiliar) vs. after training (when the objects were familiar) during both the tactile perception and memory tasks (PE and $\mathrm{MD}$ ). We hypothesized that, if the PRC does indeed represent tactile object familiarity in the blind as it does for visual object familiarity in the sighted, then we should observe the PRC's signature pattern of recognition familiarity-that is, a decrease in BOLD activation when the items become familiar (i.e., from pre-training to post-training). To ascertain whether this effect is specific to the PRC (as hypothesized) or is a more general effect that spreads to or leaks from other nearby MTL regions, we also assessed pre- to post-training differences in activation in the ERC, which is the MTL region most proximal to the PRC.

\section{MATERIALS AND METHODS}

\section{Participants}

The participants were eight blind individuals (four females, four males; ages 31-76) whose demographics are summarized in Table 1. All participants gave informed consent for the 
TABLE 1 | Participant demographics.

\begin{tabular}{|c|c|c|c|c|c|c|c|c|c|}
\hline participant \# & Gender & Age & $\begin{array}{l}\text { Current } \\
\text { visual } \\
\text { status }\end{array}$ & $\begin{array}{l}\text { Age of onset } \\
\text { of current } \\
\text { visual status }\end{array}$ & $\begin{array}{l}\text { Visual status } \\
\text { at birth }\end{array}$ & $\begin{array}{l}\text { Did participant } \\
\text { ever have full } \\
\text { vision? }\end{array}$ & $\begin{array}{c}\text { Could participant } \\
\text { ever use vision to } \\
\text { see shapes/objects? }\end{array}$ & Diagnosis & $\begin{array}{l}\text { Braille } \\
\text { fluency }\end{array}$ \\
\hline 1 & $M$ & 68 & NLP & 15 & LP & No & No & $\begin{array}{c}\text { Retinopathy of } \\
\text { prematurity }\end{array}$ & High \\
\hline 2 & $\mathrm{~F}$ & 66 & LP & $<1$ & LP & No & No & $\begin{array}{c}\text { Retinopathy of } \\
\text { prematurity }\end{array}$ & High \\
\hline 3 & $\mathrm{~F}$ & 57 & LP & 30 & Tunnel vision & No & Yes & Retinitis pigmentosa & High \\
\hline 4 & $M$ & 76 & LP & 16 & Full vision & Yes & Yes & Optic neuropathy & Moderate \\
\hline 5 & $\mathrm{~F}$ & 31 & NLP & 28 & LP & No & Yes & Optic nerve hypoplasia & High \\
\hline 6 & $\mathrm{~F}$ & 66 & NLP & 16 & Full vision & Yes & Yes & Glaucoma & Moderate \\
\hline 7 & $M$ & 70 & LP & 60 & Full vision & Yes & Yes & Optic neuropathy & None \\
\hline 8 & $M$ & 56 & LP & 47 & Full vision & Yes & Yes & Glaucoma & None \\
\hline
\end{tabular}

NLP, no light perception; LP, light perception.

experimental protocol, which was approved by the SmithKettlewell Institutional Review Board, and were compensated for their time. All participants were right-handed.

To ascertain the current visual status of all participants, the Berkeley Rudimentary Vision Test (Bailey et al., 2012) was administered during which a series of cards with black and white tumbling E's, gratings, and field projections were presented. None of the participants reported being able to perceive any of the information on these cards with either eye. Light perception was then assessed via a flashlight. Five participants had some light perception (LP) in segments of the visual field in one or in both eyes; for these participants, a blindfold was placed over their eyes for the duration of the experiment to eliminate all possible visual input. The other three participants were unable to perceive this light, thereby being classified as having no light perception (NLP). Of these NLP participants, two (\#1 and \#2) were totally blind (LP) from birth and therefore were never able to use vision to form any object representations. While our blind participants did differ with respect to their age of blindness onset, that we included these congenitally blind participants allows us to speak to whether vision is necessary in forming tactile familiarity representations in the PRC.

\section{Design}

The experimental design was as in Likova (2012, 2013, 2014; see Figure 1). In short, the key component of the study was applying the Cognitive-Kinesthetic Drawing Training as a powerful memory intervention instrument that allowed us to achieve remarkable causal changes at a behavioral level within only 5 days of $2 \mathrm{~h}$ /day sessions. Before and after completing the training, the participants were tested by fMRI. In the scanner, participants performed three tactile tasks in a block paradigm (see Figure 1A) as in Likova (2012, 2013, 2014). The three tasks were as follows: during Perceptual Exploration (PE), participants explored a raised line drawing with their left hand (a task that is predominantly perceptual, though-as any perception-it does involve memory encoding); during Memory Drawing (MD), participants used their tactile memory to draw the same image with their right hand (a non-perceptual task that requires not only access to but also implementation of the memory); and during Scribble (S), participants drew randomly with their right hand as a motor control (no perception or access to memories is needed). Each task lasted $20 \mathrm{~s}$, and participants were instructed to continue drawing/exploring for the entire $20 \mathrm{~s}$, even if they finished early, so that equal time was spent on each task. After $20 \mathrm{~s}$, the participants were told to stop what they were doing regardless of their progress. The tasks were separated by a 20 -s rest interval (RI) during which participants were instructed to clear their mind of any shapes or images. An auditory cue signaled the start of each task. The three-task sequence (RI, PE, RI, MD, RI, S, RI) was repeated twice for each line-drawing stimulus, and there were six stimuli (three faces and three objects; see Figure 1B), for a total of 12 repetitions of the sequence. Prior to beginning the pretraining fMRI session, participants were informed as to the nature of the experiment and briefly familiarized with the tasks and equipment.

During the training sessions, participants were trained on how to efficiently and accurately perform the three tasks. At the start of training, participants were allowed to explore and draw the objects without the $20 \mathrm{~s}$ time restriction while learning the detailed spatial components of the line drawings. As training progressed, the $20 \mathrm{~s}$ time limit for each task was enforced. Importantly, the same line drawings were used during training and during fMRI scanning (see Figure 1B); thus, participants became highly familiar with the set of stimuli.

\section{Equipment}

A unique custom MRI-compatible presentation and drawing system was used for the brain imaging portion of this study (see Figure 1C). The system consisted of a plexiglass table extending across the participant's lap with a dual-slot adjustable surface attached on the top. The left slot held the line drawing stimulus, while the right slot held an MRI-compatible electronic drawing tablet. Movement of the stylus (held in the participant's right hand) across this drawing tablet was recorded and presented in real-time on the control computer such that the participant's drawings were viewable to the experimenters. The auditory stimuli were presented through Resonance 
Technologies earphones (Resonance Technologies, Salem, MA).

\section{fMRI Acquisition and Analyses}

Data were collected on a Siemens Trio 3T magnet equipped with a 12-channel head coil. BOLD responses were obtained using an EPI acquisition ( $\mathrm{TR}=2 \mathrm{~s}$, TE $=28 \mathrm{~ms}$, flip angle $=80^{\circ}$, voxel size $=3.0 \mathrm{~mm} \times 3.0 \mathrm{~mm} \times 3.5 \mathrm{~mm}$ ) consisting of 35 axial slices extending across the whole brain. Pre-processing was conducted using FSL (Analysis Group, fMRIB, Oxford, UK) and included slice-time correction and two-phase motion correction, consisting of both within-scan and between-scan 6-parameter rigid-body corrections. To facilitate segmentation and registration, a whole-brain highresolution T1-weighted anatomical scan was also obtained for each participant (voxel size $=0.8 \mathrm{~mm} \times 0.8 \mathrm{~mm} \times 0.8 \mathrm{~mm}$ ). White matter segmentation in this T1 scan was conducted using FreeSurfer (Martinos Center for Biomedical Imaging, Massachusetts General Hospital) and gray matter was generated with the mrGray function in the mrVista software package (Stanford Vision and Imaging Science and Technology).

In order to obtain estimates of neural activation amplitudes for each task, a general linear model (GLM) was fit to the acquired BOLD data for each three-task sequence. The model consists of three boxcars representing the task activations plus a sequence of impulses corresponding to auditory cues convolved with an estimated hemodynamic response function (HRF), and a fourth-order polynomial for low-frequency baseline fluctuations. For each task (PE, MD, and S), statistical parametric maps (SPMs) were generated based on the estimated activation amplitudes from the above GLM in each voxel that exceeded the noise threshold defined by the variability in the residual. Voxel-wise difference $z$-score maps were also created in order to compare pre-training and post-training activation.

\section{Region of Interest Analysis}

The main region of interest (ROI) in the present study was the PRC. The left and right PRC ROIs were defined anatomically in each participant based on previously determined guidelines (Insausti et al., 1998; Kivisaari et al., 2013). Although there are often substantial individual differences in MTL anatomy, care was taken to define ROIs as consistently as possible between participants. Specifically, from anterior to posterior, the PRC was defined as beginning $2 \mathrm{~mm}$ anterior to the appearance of the limen insulae gray and ending $3 \mathrm{~mm}$ posterior to the disappearance of the ERC (see next paragraph). From medial to lateral, the PRC extended from the shoulder of the medial bank of the collateral sulcus (CS) to the shoulder of the lateral bank of the CS.

In order to ascertain that any training-induced effects we observed were specific to the PRC and not a general MTL effect, we also created left and right ROIs for the ERC. This structure, like the PRC, was defined based on pre-determined guidelines (Kivisaari et al., 2013). From anterior to posterior, the ERC was defined as beginning $2 \mathrm{~mm}$ posterior to the first appearance of the limen insulae white and ending $1 \mathrm{~mm}$ posterior to the apex of the intralimbic sulcus. From medial to lateral, the ERC extended from the medial apex of the parahippocampal gyrus to the shoulder of the medial border of the CS (i.e., up to but not extending into the $\mathrm{CS}$ ).

Using these anatomical landmarks, ROIs for the left and right PRC and ERC were hand-drawn in each individual brain in FSL (Smith et al., 2004). Figure 2 shows an example of these ROIs in the left hemisphere in one representative participant (\#2).

The Stanford package mrVista was used to estimate the neural activation amplitudes for each task within these PRC ROIs using the same GLM procedure as above, but applied to the average signal across all voxels within the ROI.

\section{RESULTS}

\section{Participant Report}

As expected, participants reported that the tactile memoryguided drawing task was quite challenging. Prior to training, some participants were not even familiar with how to properly hold a pen or with simple spatial attributes present in the raisedline drawings, such as curved vs. straight lines. When participants explored the stimuli with their left hand during PE, they initially reported (in between scans during the pre-training fMRI session) being unable to recognize the line drawings or clearly understand their detailed spatial components. When trying to draw the raised-line images from tactile memory with their right hand during MD, participants lacked confidence and often expressed conviction in the impossibility of such a task. Importantly, the use of the left hand for exploration and the right hand for drawing ensured that participants could not rely on motor memory or haptic knowledge to produce their drawings; instead, they had to rely on their memory representation of the stimulus. Moreover, by separating their two hands, participants could not employ the common strategy of using their left hand to guide the pen as they

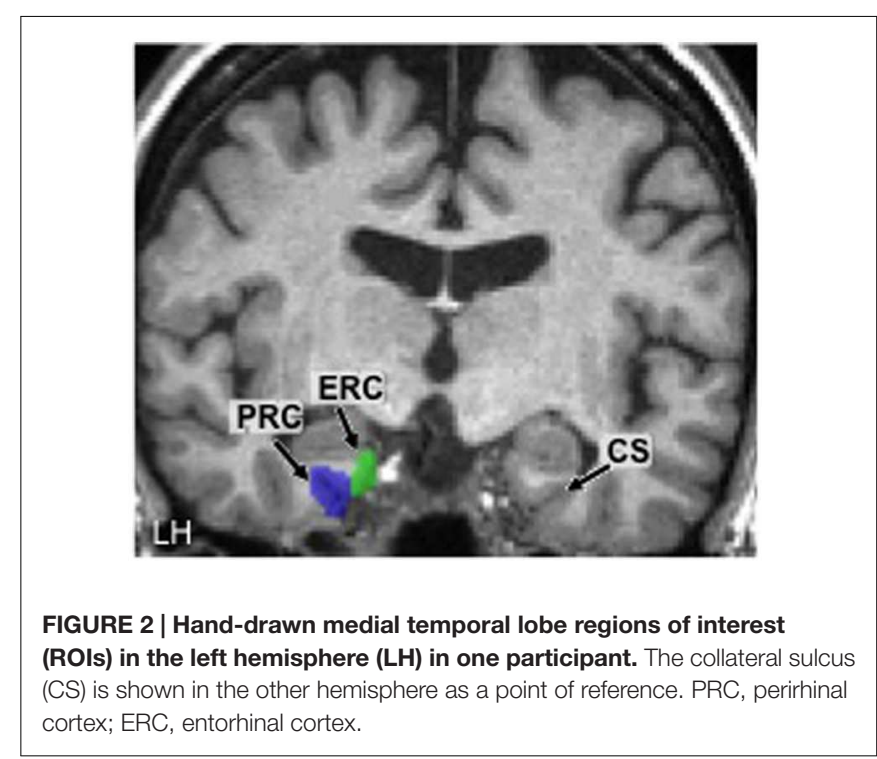


drew with their right hand, thereby making the drawing task even more challenging, as this two-hand technique is often used by blind individuals during Braille reading and writing.

After the Cognitive-Kinesthetic training, however, participants could easily recognize each stimulus during exploration, and after a 20 s rest period, could use their memory representation of the stimulus to confidently create a drawing (in only $20 \mathrm{~s}$ ) that closely resembled the original stimulus by 'projecting' it onto the drawing space on the right side of the device. Participants reported being more aware of not only the identity of each stimulus, but also the detailed spatial components that comprised it. On a more humanistic level, participants expressed certainty, happiness, and excitement with their drawings after training-a massive shift in mental and emotional state from before training, with respect to not only the task but also their self-confidence. Together, these selfreports imply that the Cognitive-Kinesthetic training allowed participants to create robust memory representations of the line-drawing stimuli, thereby rendering them familiar.

\section{Drawing Results}

Participants' memory-guided drawing ability improved substantially from pre- to post-training (see Figure 3). Drawing speed was radically enhanced, from a median value of $3 \mathrm{~min}$ per drawing at the beginning of training (when time was not restricted) to typical achievement of the complete drawing in the target time of $20 \mathrm{~s}$ or less at the end of training. Before training, drawings were disconnected, unstructured, and most often unrecognizable. After training, the drawings clearly resembled the original stimuli, consistent with participants' reports of being able to recognize the stimuli and create strong mental representations of their spatial arrangements. Individual differences were apparent in the participants' drawing abilities, as would be expected; even so, every participant's drawings improved significantly as a result of the training, even in spite of the physical limitations of the narrow bore of the scanner.

\section{fMRI Results \\ Perirhinal Cortex ROI analysis}

Results of the ROI analysis on BOLD activation in the left and right PRC are shown in Figure 4A. The data shown here represent the averages across all eight blind participants. The results show that prior to Cognitive-Kinesthetic drawing training (i.e., when the stimuli were unfamiliar), activation in the left and right PRC was significantly above zero ( $p s=0.04$ and 0.03 , respectively) during exploration of the raised-line drawing (PE task). After training, when the stimuli were familiar, PRC activation during $\mathrm{PE}$ was reduced to baseline. This pre- to post-training decrease was significant in the left PRC $(p=0.04)$ and marginally significant in the right $\operatorname{PRC}(p=0.07)$. Indeed, there was no main effect of hemisphere $(p>0.40)$, indicating that the pattern of activation did not significantly differ in the left vs. right PRC. For this reason, we collapsed the data across hemispheres (see Figure 4B). An analysis on these cross-hemisphere data

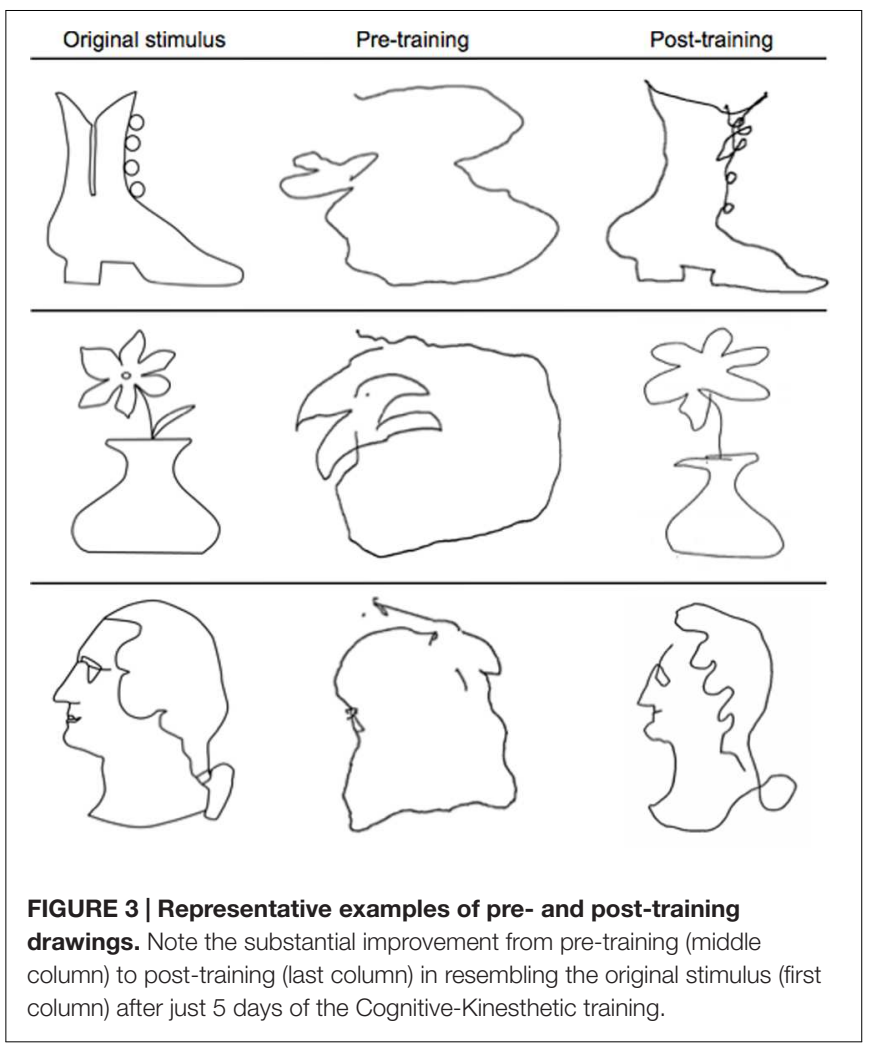

confirms a significant decrease in PRC activation from preto post-training during the $\mathrm{PE}$ task $(p=0.02)$, with pretraining activation significantly above baseline $(p=0.008)$ and post-training activation not significantly different from baseline $(p>0.70)$.

PRC activation during the MD task was much more variable both within and between participants, as can be expected for this more complex learning task of memory-guided drawing. Specifically, during MD, participants must access a detailed memory representation of the stimulus while recruiting precise motor control to produce a drawing containing detailed spatial components. Despite all of these intricacies and the resulting high variability, a significant reduction in activation from pre- to post-training was observed in both the left and right PRC ( $p$ s $=0.04$ and 0.04 , respectively) during the MD task (see Figure 4A). The BOLD responses shown here represent signals averaged across both space (all voxels within the ROI) and time (the 20-s task interval). The data collapsed across hemispheres continue to show this significant pre- to posttraining decrease in activation (see Figure $4 \mathrm{~B} ; p=0.01$ ), with post-training activation decreasing significantly below baseline $(p=0.004)$. Moreover, this pre- to post-training reduction during the $\mathrm{MD}$ task was significantly greater than the pre- to post-training difference during the $S$ control task, as evidenced by a significant session $\times$ task interaction $(p=0.03)$.

As expected, no significant differences in activation between pre- and post-training were observed during the control task (S) in either the left or right PRC. 
A

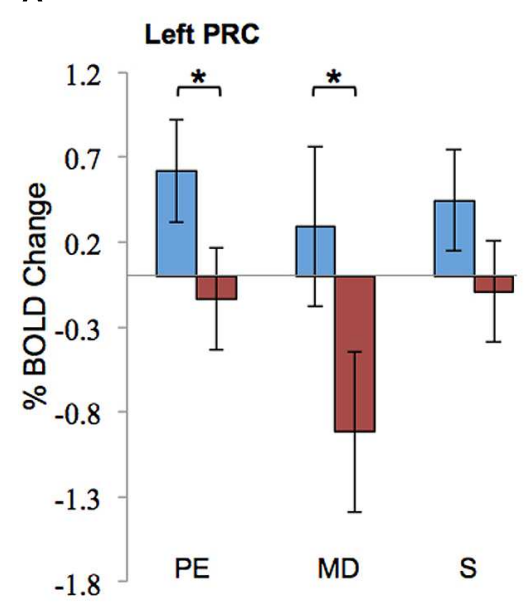

Right PRC

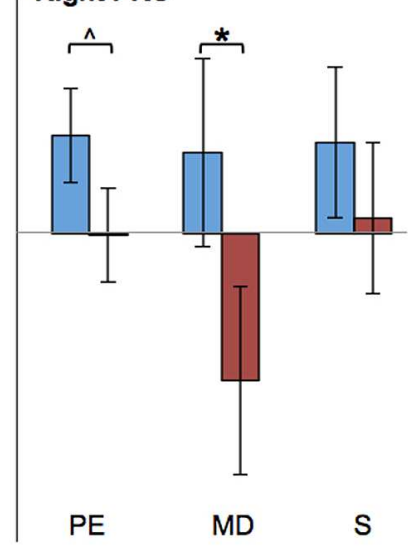

B

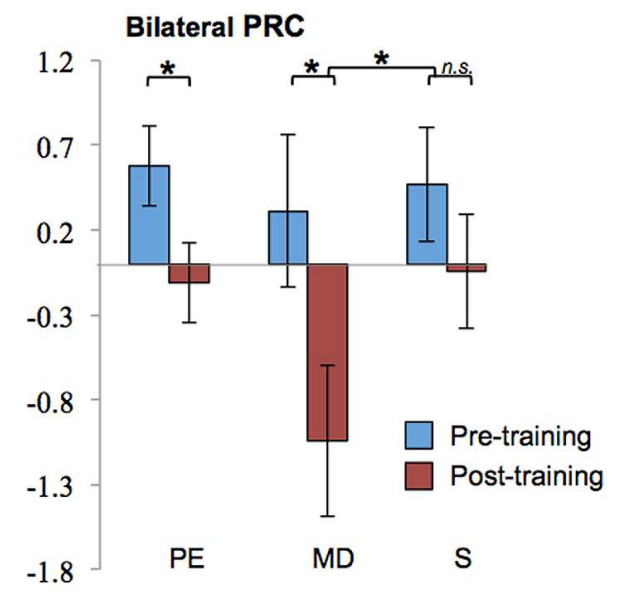

C

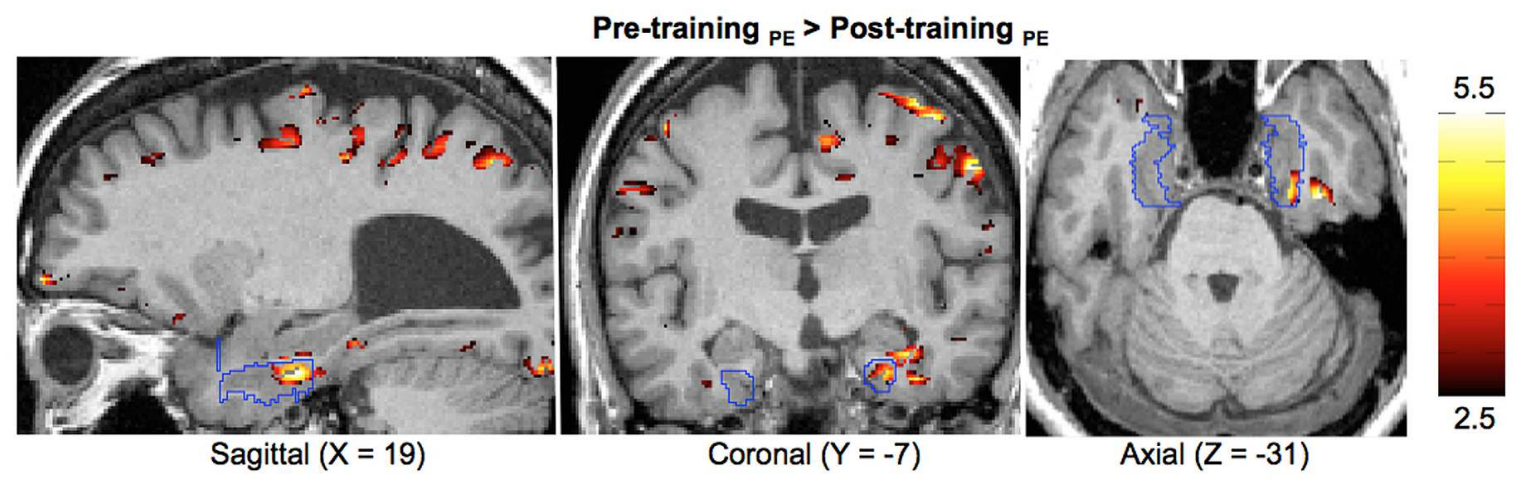

FIGURE 4 | Functional magnetic resonance imaging results. ROI analysis results for pre- and post-training are shown (A) separated by hemisphere and (B) collapsed across hemisphere. Error bars represented standard error of the mean of the difference scores (post-pre). (C) Pre-training > post-training difference $z$-score map in one participant (\#2), with the PRC ROI outlined in blue. PRC, perirhinal cortex; PE, Perceptual Exploration; MD, Memory Drawing; S, Scribble; $* p<0.05, \hat{p}<0.10$.

The main finding from the above ROI analysis is a reduction in PRC activation from pre- to post-training during perceptual exploration (PE task) and memory-guided drawing (MD task) of the line drawing stimuli across all participants, consistent with their becoming familiar with the stimuli by the repeated exposure during the training period.

\section{Cluster analysis}

To ascertain the robustness of this familiarity-based reduction in PRC activation in each individual participant, we also generated difference $z$-score maps comparing pre- vs. post-training to perform a cluster analysis. In every participant, a cluster of significant voxel activation differences $(z>1.96, p<0.05$ per voxel) was found in both the left and right PRC during the PE task in the direction of pre-training $>$ post-training (see Table 2; Figure 4C depicts this significant cluster in the right hemisphere in one representative participant). This significant familiaritybased reduction in activation from pre- to post-training during $\mathrm{PE}$ in each participant is consistent with the above analysis collapsed across participants. That the effect is observable both within and across participants speaks to its robustness, despite the minor variations in location of the maximum familiarity-based reduction.

The cluster analysis also revealed a suprathreshold cluster of significant voxel activation differences $(z>1.96, p<0.05$ per voxel) in the direction of pre-training $>$ post-training during the MD task in the left PRC in every participant and in the right PRC for seven out of the eight participants. This result is consistent with the significance observed in the ROI analysis across participants during the MD task.

Together, these results indicate that the familiarity-based reduction in activation observed in the PRC during PE (a predominately perceptual task) is also apparent during $\mathrm{MD}$ (a non-perceptual memory task). This finding in the blind coincides with previous work in the sighted showing that the $\mathrm{PRC}$ is involved in familiarity representations that subserve both perception and memory.

\section{Entorhinal Cortex}

In order to ascertain that our familiarity-based reduction in activation was an effect specific to the PRC, we conducted ROI analyses on the immediately adjacent ERC ROI as well. As in 
TABLE 2 | Clusters in the PRC showing significant pre-training $>$ post-training differences.

\begin{tabular}{lllll}
\hline Participant \# & \multicolumn{3}{c}{ Left } & \multicolumn{2}{l}{ Right } \\
\cline { 2 - 5 } & Max Z & Peak coordinates & Max Z & Peak coordinates \\
\hline PE task & & & & \\
1 & 2.25 & $-31,-8,-25$ & 3.01 & $33,-13,-36$ \\
2 & 3.84 & $-28,-14,-22$ & 5.6 & $21,-16,-27$ \\
3 & 3.31 & $-27,-8,-25$ & 2.44 & $32,6,-31$ \\
4 & 6.87 & $-31,-20,-23$ & 5.52 & $35,-24,-29$ \\
5 & 3.15 & $-27,-6,-26$ & 2.91 & $21,-27,-17$ \\
6 & 6.22 & $-30,-10,-23$ & 4.7 & $25,-9,-22$ \\
7 & 2.40 & $-30,-6,-26$ & 2.12 & $40,-18,-22$ \\
8 & 8.33 & $-33,-11,-33$ & 8.91 & $33,-9,-33$ \\
MD task & & & & \\
1 & 2.76 & $-31,-16,-22$ & - & \\
2 & 3.5 & $-28,-13,-26$ & 3.2 & $23,-14,-25$ \\
3 & 4.69 & $-27,-8,-26$ & 5.23 & $30,-10,-27$ \\
4 & 5.39 & $-32,-18,-24$ & 2.28 & $26,-10,-26$ \\
5 & 3.05 & $-36,-25,-20$ & 3.08 & $33,-22,-26$ \\
6 & 3.56 & $-30,-8,-24$ & 7.17 & $26,-6,-26$ \\
7 & 2.63 & $-36,-15,-29$ & 3.65 & $36,-9,-26$ \\
8 & 8.53 & $-33,-12,-32$ & 9.41 & $34,-11,-22$ \\
\hline
\end{tabular}

$P E$, Perceptual Exploration, MD, Memory Drawing; coordinates are in Talairach space $(x, y, z)$.

the PRC, there was no main effect of hemisphere in this area ( $p s>0.30$ ), allowing us to collapse the data across left and right ROIs. However, in contrast to the PRC, analyses on the ERC data produced no significant differences between pre-training and post-training activation in any of the three task conditions (PE, $\mathrm{MD}$, or $S), p s>0.20$. The lack of a pre- to post-training difference in the ERC could suggest that our tactile familiarity effect is restricted to the PRC, or that the weaker involvement of the ERC in recognition was not sufficient to produce a measureable difference in activation. Future research could elucidate the ERC's role in tactile object familiarity.

\section{DISCUSSION}

This study is the first to examine the ability of the PRC to represent tactile-rather than visual-object familiarity in the blind. Furthermore, it investigates familiarity representations during both perception and memory. "Familiarity" in this sense refers to the prior exposure to an object such that the object is meaningful and recognizable to the observer. Previous work in the sighted shows that the signature pattern of PRC activation in representing object familiarity is a decrease in neural activity when an item is made experimentally familiar (Ringo, 1996; Brown and Xiang, 1998; Suzuki and Eichenbaum, 2000; Brown and Aggleton, 2001; Henson et al., 2003). This effect has been observed in the visual modality for both memory and perception. While there is some prior evidence of cross-modal representations in the PRC (Desimone and Gross, 1979; Suzuki et al., 1993; Holdstock et al., 2009; Martin et al., 2011), evidence of tactile representations per se is limited and is restricted to memory only. Furthermore, the PRC's involvement in tactile memory has previously only been investigated in the sighted. Thus, it remains unclear whether vision is necessary in establishing familiarity representations in the PRC, and whether there are supramodal familiarity representations for not memory only but perception as well.

Here, we used a unique fMRI learning paradigm to show that the PRC represents tactile object familiarity in the blind, including those who have never had full vision. Specifically, after 5 days of the Cognitive-Kinesthetic training (Likova, 2010, 2012, 2013, 2014) during which participants became able to perform memory-guided blind drawing and became familiar with a set of raised-line drawings of faces and objects, PRC activation significantly decreased bilaterally in response to increased familiarity with the stimuli. This familiarity-based reduction was observed in the group analysis as well as the individual cluster analysis, thereby speaking to the robustness of this pre- to post-training decline. Thus, the results from this study show for the first time that the PRC represents tactile object familiarity in the blind, and that these representations can form independently from visual experience. By revealing that the PRC can reorganize to represent tactile information when necessary, this work has important implications for the fields of perception and memory as well as for the field of blindness rehabilitation.

The familiarity-based reduction in PRC activation in the present study was observed during PE when participants were exploring the raised-line drawings (a task that is predominantly perceptual, but does involve access to and encoding of object memories) and during MD when they were drawing the images from tactile memory (a non-perceptual memory retrieval task). This finding is consistent with recent work in the visual modality which has shown that the PRC's familiarity representations subserve both memory and perception (Murray and Bussey, 1999; Bussey et al., 2002; Murray et al., 2007; Baxter, 2009; Peterson et al., 2012; Nadel and Peterson, 2013). For instance, the PRC has been implicated in visual recognition memory (Ringo, 1996; Brown and Xiang, 1998; Suzuki and Eichenbaum, 2000; Brown and Aggleton, 2001; Henson et al., 2003) and also in visual familiarity/novelty discrimination when two objects are presented simultaneously (Ennaceur et al., 1996; Ennaceur and Aggleton, 1997; Abe et al., 2004). During such perceptual discriminations, existing stored familiarity representations must be accessed and matched to the currently perceived visual input. Likewise, in the current study, when participants tactually explore the raised-line drawings during PE, they are attempting to match the perceived stimulus to an existing familiarity representationin this case, one created via the tactile modality. Similarly, during $\mathrm{MD}$, the existing familiarity representations must be accessed in detail in order to guide drawing. In this way, the familiarity representations created during the CognitiveKinesthetic training are used to facilitate both PE and MD. As such, both of these tasks rely on access to memories; the difference lies in the reason for access and how that memory is utilized.

Overall, the results of the present tactile perception and memory study show for the first time that the PRC is not only 
perceptual-mnemonic in nature-as in the visual modality-but moreover, since these familiarity effects were found in the tactile modality, that the PRC's perceptual and mnemonic representations are supramodal.

The finding of supramodality in the PRC in the present study adds to previous work revealing the supramodal nature of other cortical areas, as assessed in blind individuals. For instance, Likova $(2010,2012,2013)$ has shown that low-level "visual" regions, including V1 and extrastriate cortex, can reorganize to represent tactile information after just 5 days of the Cognitive-Kinesthetic training. Even without this training, the blind visual cortex is active during certain tactile and auditory tasks, including reading, discriminating Braille (Sadato et al., 1996, 2002) and hearing echoes produced during echolocation (Thaler et al., 2011). Higher in the representational hierarchy, the inferotemporal cortex and even the hippocampus have been shown to undergo training-induced reorganization toward tactile representations in the blind as well (Likova, 2015). Additionally, areas of the parietal cortex typically implicated in visual spatial attention-including the intraparietal sulcus and superior parietal lobule-have also been shown to be supramodal in nature (Leo et al., 2012; Ricciardi et al., 2014). Moreover, these parietal areas have strong functional connectivity with the visual cortex during tactile perception (Leo et al., 2012), further pointing toward the supramodality of not only individual cortical regions but also the networks in which they are involved. The current study goes further to demonstrate that such a key MTL region as the PRC is also a supramodal structure, with its object familiarity representations apparent in the tactile domain. This work, along with the previous literature, indicates that visual experience is not necessary in forming spatial representations.

It is interesting to consider whether the familiarity-based decrease in PRC activation observed in the present study is due to familiarity at the perceptual or semantic level. Previous research has shown that the PRC indeed represents highlevel semantic information pertaining to objects (Bruffaerts et al., 2013; Clarke and Tyler, 2014) such that the assessment of an object's meaningfulness is impaired when the PRC is compromised, as in semantic dementia (Barense et al., 2010b). Our definition of "familiarity" in the present study is very closely related to (if not synonymous with) "meaningfulness"; thus, our observed familiarity effects are likely at the level of semantic familiarity. That we found our familiarity effect during the MD task where participants must rely on mental representations rather than immediate perceptions supports this semantic-level familiarity. Of course, participants did develop a familiarity with both perceptual and semantic components of the stimuli over the course of the training, and given the PRC's involvement in both perception and memory (e.g., Murray and Bussey, 1999; Bussey et al., 2002, 2005; Murray et al., 2007; Baxter, 2009; Peterson et al., 2012; Nadel and Peterson, 2013), it likely represents familiarity at multiple levels.

Previous research on the PRC (in the visual modality) has not only demonstrated its involvement in representing object familiarity, but also in representing objects comprised of complex conjunctions of features (Bussey et al., 2005). For instance, neurophysiological research has demonstrated that monkeys and rats with selective lesions to the PRC are impaired on visual discrimination tasks when the objects possess a high degree of feature ambiguity-that is, when the objects have multiple features in common (Bussey and Saksida, 2002; Bussey et al., 2002; Bartko et al., 2007). Similarly, humans with damage to the MTL including the PRC are impaired at object discrimination under conditions of high feature ambiguity, whereas humans with damage restricted to the hippocampus do not exhibit this deficit (Lee et al., 2005; Barense et al., 2007). FMRI studies have provided further supporting evidence for the PRC's involvement in feature complexity/ambiguity, showing that PRC activity is higher during a discrimination task when the objects possess a high vs. low degree of feature ambiguity, even when controlling for level of difficulty (Barense et al., 2010a). To date, no studies have investigated this feature-conjunction model of PRC function using tactile stimuli or in those who are blind. In the present study, we did not directly manipulate feature complexity or ambiguity of our tactile raised-line drawing stimuli. However, all of our line drawings could be considered complex tactile stimuli, as they depicted whole, real-world objects comprised of many complex features (eyes, hairlines, petals, handles, etc.; see Figure 1B). In designing our stimuli this way, we may have increased the probability of PRC involvement in representing each individual stimulus. Likewise, that we found PRC involvement for these complex stimuli support feature conjunction models of PRC function (Bussey and Saksida, 2002; Cowell et al., 2006). Across our set of stimuli, the tactile line drawings did possess some degree of feature ambiguity; for instance, the faces all were comprised of eyes, noses, and chins. Thus, it is possible that the PRC was recruited (during both $\mathrm{PE}$ and $\mathrm{MD}$ ) in order to differentiate between the highly similar and complex stored representations across these stimuli. Separate studies would need to be conducted in order to fully understand how the blind PRC represents tactile objects with differing degrees of feature ambiguity and complexity.

Although our raised-line drawing stimuli did include both faces and objects, we did not have the statistical power needed to conduct an analysis comparing the two categories given our small set of stimuli, number of repetitions, and number of participants. The PRC has been shown to respond to many different categories of complex objects, including faces (Barense et al., 2010a), objects (Ennaceur and Aggleton, 1997; Bussey et al., 2002; Lee et al., 2005; Barense et al., 2007, 2010b; Bartko et al., 2007), words (Bruffaerts et al., 2013), and even meaningless stimuli like blobs and "greebles" (Barense et al., 2007, 2010b). Thus, had we been able to conduct a categorical analysis, we might not have expected to observe a difference between faces and objects in the PRC. Other areas of the inferior temporal cortex, though, have been shown to differentiate between stimulus categories (e.g., Kriegeskorte et al., 2008; Clarke and Tyler, 2014). Future research should elucidate differences in activation between different object categories along the visual hierarchy in the blind. 


\section{CONCLUSION}

The results of the present study show for the first time that the PRC represents tactile object familiarity in humans, and particularly in blind individuals. Furthermore, our finding of object familiarity effects in the PRC in both tactile perception and tactile memory in blindness provides the critical link to the establishment of the PRC as a supramodal perceptual-mnemonic brain structure. Future research could further explore the underlying architectural principles and familiarity-related role of other MTL structures, such as the ERC, in non-visual perception and memory in the blind.

\section{AUTHOR CONTRIBUTIONS}

LC and LL both contributed to the idea, participant recruitment, implementation, data analysis, and writing of the manuscript.

\section{REFERENCES}

Abe, H., Ishida, Y., and Iwasaki, T. (2004). Perirhinal N-methyl-D-aspartate and muscarinic systems participate in object recognition in rats. Neurosci. Lett. 356, 191-194. doi: 10.1016/j.neulet.2003.11.049

Amedi, A., Jacobson, G., Hendler, T., Malach, R., and Zohary, E. (2002). Convergence of visual and tactile shape processing in the human lateral occipital complex. Cereb. Cortex 12, 1202-1212. doi: 10.1093/cercor/12.11.1202

Bailey, I. L., Jackson, A. J., Minto, H., Greer, R. B., and Chu, M. A. (2012). The Berkeley rudimentary vision test. Optom. Vis. Sci. 89, 1257-1264. doi: 10.1097/OPX.0b013e318264e85a

Barense, M. D., Gaffan, D., and Graham, K. S. (2007). The human medial temporal lobe processes online representations of complex objects. Neuropsychologia 45 , 2963-2974. doi: 10.1016/j.neuropsychologia.2007.05.023

Barense, M. D., Henson, R. N., Lee, A. C., and Graham, K. S. (2010a). Medial temporal lobe activity during complex discrimination of faces, objects, and scenes: effects of viewpoint. Hippocampus 20, 389-401. doi: 10.1002/hipo. 20641

Barense, M. D., Rogers, T. T., Bussey, T. J., Saksida, L. M., and Graham, K. S. (2010b). Influence of conceptual knowledge on visual object discrimination: insights from semantic dementia and MTL amnesia. Cereb. Cortex 20, 25682582. doi: 10.1093/cercor/bhq004

Bartko, S. J., Winters, B. D., Cowell, R. A., Saksida, L. M., and Bussey, T. J. (2007). Perceptual functions of perirhinal cortex in rats: zero-delay object recognition and simultaneous oddity discriminations. J. Neurosci. 27, 2548-2559. doi: 10.1523/JNEUROSCI.5171-06.2007

Baxter, M. G. (2009). Involvement of medial temporal lobe structures in memory and perception. Neuron 61, 667-677. doi: 10.1016/j.neuron.2009.02.007

Brown, M. W., and Aggleton, J. P. (2001). Recognition memory: what are the roles of the perirhinal cortex and hippocampus? Nat. Rev. Neurosci. 2, 51-61. doi: $10.1038 / 35049064$

Brown, M. W., and Xiang, J. Z. (1998). Recognition memory: neuronal substrates of the judgement of prior occurrence. Prog. Neurobiol. 55, 149-189. doi: 10.1016/S0301-0082(98)00002-1

Bruffaerts, R., Dupont, P., Peeters, R., De Deyne, S., Storms, G., and Vandenberghe, R. (2013). Similarity of fMRI activity patterns in left perirhinal cortex reflects semantic similarity between words. J. Neurosci. 33, 18597-18607. doi: 10.1523/JNEUROSCI.1548-13.2013

Buffalo, E. A., Reber, P. J., and Squire, L. R. (1998). The human perirhinal cortex and recognition memory. Hippocampus 8, 330-339. doi: 10.1002/(SICI)10981063(1998)8:4<330::AID-HIPO3>3.3.CO;2-2
LL developed the experimental design, the Cognitive-Kinesthetic method used to train the blind people to draw, and conducted the training of each participant.

\section{FUNDING}

This work was supported by the National Eye Institute at the National Institute of Health (R01EY024056), awarded to LL. LC was supported by a Rachel C. Atkinson Postdoctoral Fellowship while working on this study.

\section{ACKNOWLEDGMENTS}

The authors would like to thank Spero Nicholas for helping with data collection and analysis, Kristyo Mineff for assisting with the drawing training, and Christopher W. Tyler for a careful review of the manuscript.

Bussey, T. J., and Saksida, L. M. (2002). The organization of visual object representations: a connectionist model of effects of lesions in perirhinal cortex. Eur. J. Neurosci. 15, 355-364. doi: 10.1046/j.0953-816x.2001.01850.x

Bussey, T. J., Saksida, L. M., and Murray, E. A. (2002). Perirhinal cortex resolves feature ambiguity in complex visual discriminations. Eur. J. Neurosci. 15, 365-374. doi: 10.1046/j.0953-816x.2001.01851.x

Bussey, T. J., Saksida, L. M., and Murray, E. A. (2005). The perceptualmnemonic/feature conjunction model of perirhinal cortex function. Q. J. Exp. Psychol.-B 58, 269-282. doi: 10.1080/02724990544000004

Cowell, R. A., Bussey, T. J., and Saksida, L. M. (2006). Why does brain damage impair memory? A connectionist model of object recognition memory in perirhinal cortex. J. Neurosci. 26, 12186-12197. doi: 10.1523/JNEUROSCI. 2818-06.2006

Clarke, A., and Tyler, L. K. (2014). Object-specific semantic coding in human perirhinal cortex. J. Neurosci. 34, 4766-4775. doi: 10.1523/JNEUROSCI.282813.2014

Desimone, R., and Gross, C. G. (1979). Visual areas in the temporal cortex of the macaque. Brain Res. 178, 363-380. doi: 10.1016/0006-8993(79)90699-1

Ennaceur, A., and Aggleton, J. P. (1997). The effects of neurotoxic lesions of the perirhinal cortex combined to fornix transection on object recognition memory in the rat. Behav. Brain Res. 88, 181-193. doi: 10.1016/S0166-4328(97)0 2297-3

Ennaceur, A., and Delacour, J. (1988). A new one-trial test for neurobiological studies of memory in rats. 1: behavioral data. Behav Brain Res. 31, 47-59. doi: 10.1016/0166-4328(88)90157-X

Ennaceur, A., Neave, N., and Aggleton, J. P. (1996). Neurotoxic lesions of the perirhinal cortex do not mimic the behavioural effects of fornix transection in the rat. Behav. Brain Res. 80, 9-25. doi: 10.1016/0166-4328(96)00006-X

Henson, R. N. A., Cansino, S., Herron, J. E., Robb, W. G. K., and Rugg, M. D. (2003). A familiarity signal in human anterior medial temporal cortex? Hippocampus 13, 301-304. doi: 10.1002/hipo.10117

Holdstock, J. S., Hocking, J., Notley, P., Devlin, J. T., and Price, C. J. (2009). Integrating visual and tactile information in the perirhinal cortex. Cereb. Cortex 19, 2993-3000. doi: 10.1093/cercor/bhp073

Insausti, R., Juottonen, K., Soininen, H., Insausti, A. M., Partanen, K., Vainio, P., et al. (1998). MR volumetric analysis of the human entorhinal, perirhinal, and temporopolar cortices. Am. J. Neuroradiol. 19, 659-671.

Kivisaari, S. L., Probst, A., and Taylor, K. I. (2013). "The perirhinal, entorhinal, and parahippocampal cortices and hippocampus: an overview of functional anatomy and protocol for their segmentation in MR images," in fMRI: Basics Clinical Application, eds S. Ulmer and O. Jansen (Berlin: Springer), 239-267. 
Kriegeskorte, N., Mur, M., Ruff, D. A., Kiani, R., Bodurka, J., Esteky, H., et al. (2008). Matching categorical object representations in inferior temporal cortex of man and monkey. Neuron 60, 1126-1141. doi: 10.1016/j.neuron.2008.10.043

Lee, A. C., Buckley, M. J., Pegman, S. J., Spiers, H., Scahill, V. L., Gaffan, D., et al. (2005). Specialization in the medial temporal lobe for processing of objects and scenes. Hippocampus 15, 782-797. doi: 10.1002/hipo.20101

Leo, A., Bernardi, G., Handjaras, G., Bonino, D., Ricciardi, E., and Pietrini, P. (2012). Increased BOLD variability in the parietal cortex and enhanced parieto-occipital connectivity during tactile perception in congenitally blind individuals. Neural Plast. 2012, 8. doi: 10.1155/2012/720278

Likova, L. T. (2010). "Drawing in the blind and the sighted as a probe of cortical reorganization," in IS\&T/SPIE Electronic Imaging, eds. B. E. Rogowitz and T. N. Pappas (Orlando, FL: International Society for Optics and Photonics), 752708-752720.

Likova, L. T. (2012). Drawing enhances cross-modal memory plasticity in the human brain: a case study in a totally blind adult. Front. Hum. Neurosci. 6:44. doi: 10.3389/fnhum.2012.00044

Likova, L. T. (2013). A cross-modal perspective on the relationships between imagery and working memory. Front. Psychol. 3:561. doi: 10.3389/fpsyg.2012.00561

Likova, L. T. (2014). "Learning-based cross-modal plasticity in the human brain: insights from visual deprivation fMRI," in Advanced Brain Neuroimaging Topics in Health and Disease - Methods and Applications, Chap. 13, eds T. D. Papageorgiou, G. I. Christopoulos, and S. M. Smirnakis (Rijeka: Intech Press), 327-358.

Likova, L. T. (2015). “Temporal evolution of brain reorganization under crossmodal training: insights into the functional architecture of encoding and retrieval networks," in IS\&T/SPIE Electronic Imaging, Vol. 9394, eds. B. E. Rogowitz, H. deRidder, and T. N. Pappas (Orlando, FL: International Society for Optics and Photonics), 939417-939432.

Martin, C. B., Bowles, B., Mirsattari, S. M., and Köhler, S. (2011). Selective familiarity deficits after left anterior temporal-lobe removal with hippocampal sparing are material specific. Neuropsychologia 49, 1870-1878. doi: 10.1016/j.neuropsychologia.2011.03.012

Meunier, M., Bachevalier, J., Mishkin, M., and Murray, E. A. (1993). Effects on visual recognition of combined and separate ablations of the entorhinal and perirhinal cortex in rhesus monkeys. J. Neurosci. 13, 5418-5432.

Mumby, D. G., and Pinel, J. P. (1994). Rhinal cortex lesions and object recognition in rats. Behav. Neurosci. 108, 11. doi: 10.1037/0735-7044.108.1.11

Murray, E. A., and Bussey, T. J. (1999). Perceptual-mnemonic functions of the perirhinal cortex. Trends Cogn Sci. 3, 142-151. doi: 10.1016/S13646613(99)01303-0

Murray, E. A., Bussey, T. J., and Saksida, L. M. (2007). Visual perception and memory: a new view of medial temporal lobe function in primates and rodents. Annu. Rev. Neurosci. 30, 99-122. doi: 10.1146/annurev.neuro.29.051605.113046

Murray, E. A., and Richmond, B. J. (2001). Role of perirhinal cortex in object perception, memory, and associations. Curr. Opin. Neurobiol. 11, 188-193. doi: 10.1016/S0959-4388(00)00195-1

Nadel, L., and Peterson, M. A. (2013). The hippocampus: Part of an interactive posterior representational system spanning perceptual and memorial systems. J. Exp. Psychol. Gen. 142, 1242. doi: 10.1037/a0033690

Pascual-Leone, A., Amedi, A., Fregni, F., and Merabet, L. B. (2005). The plastic human brain cortex. Annu. Rev. Neurosci. 28, 377-401. doi: 10.1146/annurev.neuro.27.070203.144216
Peterson, M. A., Cacciamani, L., Barense, M. D., and Scalf, P. E. (2012). The perirhinal cortex modulates V2 activity in response to the agreement between part familiarity and configuration familiarity. Hippocampus 22, 1965-1977. doi: 10.1002/hipo. 22065

Ricciardi, E., Bonino, D., Pellegrini, S., and Pietrini, P. (2014). Mind the blind brain to understand the sighted one! Is there a supramodal cortical functional architecture? Neurosci. Biobehav. Rev. 41, 64-77. doi: 10.1016/j.neubiorev.2013.10.006

Ringo, J. L. (1996). Stimulus specific adaptation in inferior temporal and medial temporal cortex of the monkey. Behav. Brain Res. 76, 191-197. doi: 10.1016/0166-4328(95)00197-2

Sadato, N., Okada, T., Honda, M., and Yonekura, Y. (2002). Critical period for cross-modal plasticity in blind humans: a functional MRI study. Neuroimage 16, 389-400. doi: 10.1006/nimg.2002.1111

Sadato, N., Pascual-Leone, A., Grafman, J., Ibañez, V., Deiber, M. P., Dold, G., et al. (1996). Activation of the primary visual cortex by Braille reading in blind subjects. Nature 380, 526-528. doi: 10.1038/380526a0

Sathian, K., and Stilla, R. (2010). Cross-modal plasticity of tactile perception in blindness. Restor. Neurol. Neurosci. 28, 271-281. doi: 10.3233/RNN-201 0-0534

Smith, S. M., Jenkinson, M., Woolrich, M. W., Beckmann, C. F., Behrens, T. E., Johansen-Berg, H., et al. (2004). Advances in functional and structural MR image analysis and implementation as FSL. Neuroimage 23, S208-S219. doi: 10.1016/j.neuroimage.2004.07.051

Suzuki, W. A., and Amaral, D. G. (1994). Perirhinal and parahippocampal cortices of the macaque monkey: cortical afferents. J. Comp. Neurol. 350, 497-533. doi: $10.1002 /$ cne. 903500402

Suzuki, W. A., and Eichenbaum, H. (2000). The neurophysiology of memory. Ann. N. Y. Acad. Sci. 911, 175-191. doi: 10.1111/j.1749-6632.2000.tb06726.x

Suzuki, W. A., Zola-Morgan, S., Squire, L. R., and Amaral, D. G. (1993). Lesions of the perirhinal and parahippocampal cortices in the monkey produce longlasting memory impairment in the visual and tactual modalities. J. Neurosci. 13, 2430-2451.

Taylor, K. I., Moss, H. E., Stamatakis, E. A., and Tyler, L. K. (2006). Binding crossmodal object features in perirhinal cortex. Proc. Natl. Acad. Sci. U.S.A. 103, 8239-8244. doi: 10.1073/pnas.0509704103

Thaler, L., Arnott, S. R., and Goodale, M. A. (2011). Neural correlates of natural human echolocation in early and late blind echolocation experts. PLOS ONE 6:e20162. doi: 10.1371/journal.pone.0020162

Zola-Morgan, S., Squire, L. R., Amaral, D. G., and Suzuki, W. A. (1989). Lesions of perirhinal and parahippocampal cortex that spare the amygdala and hippocampal formation produce severe memory impairment. J. Neurosci. 9, 4355-4370.

Conflict of Interest Statement: The authors declare that the research was conducted in the absence of any commercial or financial relationships that could be construed as a potential conflict of interest.

Copyright (c) 2016 Cacciamani and Likova. This is an open-access article distributed under the terms of the Creative Commons Attribution License (CC BY). The use, distribution or reproduction in other forums is permitted, provided the original author(s) or licensor are credited and that the original publication in this journal is cited, in accordance with accepted academic practice. No use, distribution or reproduction is permitted which does not comply with these terms. 